Revue des patrimoines

\title{
Abbayes, couvents et monastères devenus dépôts d'étalons impériaux, royaux et nationaux
}

\section{Tanneguy de Sainte Marie}

\section{(2) OpenEdition}

\section{Journals}

Édition électronique

URL : http://journals.openedition.org/insitu/9646

DOI : 10.4000/insitu.9646

ISSN : 1630-7305

\section{Éditeur}

Ministère de la culture

Référence électronique

Tanneguy de Sainte Marie, «Abbayes, couvents et monastères devenus dépôts d'étalons impériaux, royaux et nationaux », In Situ [En ligne], 18 | 2012, mis en ligne le 31 juillet 2012, consulté le 03 mai 2019. URL : http://journals.openedition.org/insitu/9646 ; DOI : 10.4000/insitu.9646

Ce document a été généré automatiquement le 3 mai 2019.

\section{(c) $(5)$}

In Situ Revues des patrimoines est mis à disposition selon les termes de la licence Creative Commons Attribution - Pas d'Utilisation Commerciale - Pas de Modification 4.0 International. 


\title{
Abbayes, couvents et monastères devenus dépôts d'étalons impériaux, royaux et nationaux
}

\author{
Tanneguy de Sainte Marie
}

1 Pour accueillir, héberger, dresser, exercer, travailler, présenter des chevaux et loger les hommes qui les choyaient, de tout temps il a fallu construire des arènes, des cirques et donc des écuries. Plus près de nous, le XVII ${ }^{e}$ siècle leur a construit des palais, le XVIII ${ }^{e}$ siècle des cours, le XIX ${ }^{e}$ siècle des quartiers, le $\mathrm{XX}^{\mathrm{e}}$ siècle des sites et des pôles. Le XXI siècle naissant lui dédie, à nouveau, des stades, des parcs et des complexes.

2 Quoi qu'il en soit, le cheval, cet animal sauvage contenu par l'homme et, aujourd'hui, surtout par la femme, lui impose, au quotidien, des contraintes, des astreintes, des nuits blanches, des aigreurs et autres esclavages appelés « loisirs». Mais que deviennent nos écuries de pierre où les étalons de l'État servaient les stratégies militaires?

Besançon, Blois, Corbigny, Langonnet, Le Bec-Hellouin, Montier-en-Der, Saint-Lô, Villeneuve-sur-Lot, Aurillac, Rodez, Cluny, Charleville, Annecy, Angers, Braisne, Caen, Saintes, Guingamp et bien d'autres... Les effets et les conséquences de la Révolution française de 1789 ont entraîné la chasse aux religieux et la confiscation des monastères, couvents, abbayes, chapelles et oratoires.

4 Ces immeubles, devenus biens nationaux ou communaux, étaient voués à être exploités en carrières de pierres dans leur très grande majorité lorsque, entre 1797 et 1814, le Général Bonaparte, devenu Premier Consul puis l'Empereur Napoléon I ${ }^{\mathrm{er}}$ en fit investir (et sauver!) une douzaine pour y installer des dépôts d'étalons et autant d'autres pour y loger des dépôts de remonte militaire.

5 Après visites et expertises de certains sites, il fut très vite décidé qu'un cloître pouvait servir de manège en son centre et de cheminement couvert pour promener les étalons en main en périphérie, voire de boxes en le cloisonnant et en installant des portes dans les arcades. Qu'un réfectoire équipé de bas-flancs devenus stalles pouvait accueillir une 
vingtaine de chevaux. Que l'église, qui avait été débarrassée de son mobilier et de ses ornements, pouvait servir de grange à fourrage et d'écurie. Que les cellules des moines serviraient de chambres aux palefreniers et le logis des hôtes de logements pour le directeur et les officiers. Les prairies, les bois, les jardins, les puits, les étangs, la rivière et les annexes assureront les compléments d'intendance.

Déjà à Besançon dès 1754, après le rattachement de la Franche-Comté à la France, un premier dépôt d'étalons avait été aménagé dans l'ancien couvent des Dominicains. L'église était équipée de cinq stalles dans l'abside, d'une réserve à fourrage dans le chœur, de deux fois 14 stalles dans la nef et d'une infirmerie de 10 stalles dans l'entrée et le bas de l'édifice. Chacun de ces quatre locaux cloisonnés était judicieusement aménagé dans le travers du bâtiment avec des portes indépendantes percées dans les murs des bas-côtés. Cet établissement, vieillissant et à l'étroit, sera déplacé à Pontarlier, puis à Jussey et enfin dans le quartier de la Butte à Besançon en 1852, sur son site actuel. Agrandi en 1914, incendié en 1946, il sera reconstruit en 1952.

7 En 1802, Napoléon fait transformer une partie de l'abbaye du Bec-Hellouin (SeineMaritime) en un dépôt d'étalons, dépendant du Haras du Pin (fig. $\mathbf{n}^{\circ}{ }^{\circ}$ ). Il abritera jusqu'à quelque cinquante étalons. On installa des stalles dans l'église abbatiale et dans le réfectoire mauriste qui, du coup, deviendra la grande écurie. L'intérieur du cloître du XVII siècle servira de manège, ses arcades seront cloisonnées et transformées en boxes. On y adjoindra en 1831, dans d'autres locaux, une succursale du dépôt de remonte militaire de Caen.

8 Après la fermeture du dépôt d'étalons puis du dépôt de remonte militaire du Bec Hellouin, ce qui restera de l'abbaye sera définitivement rendu à des religieux à partir de 1947. 

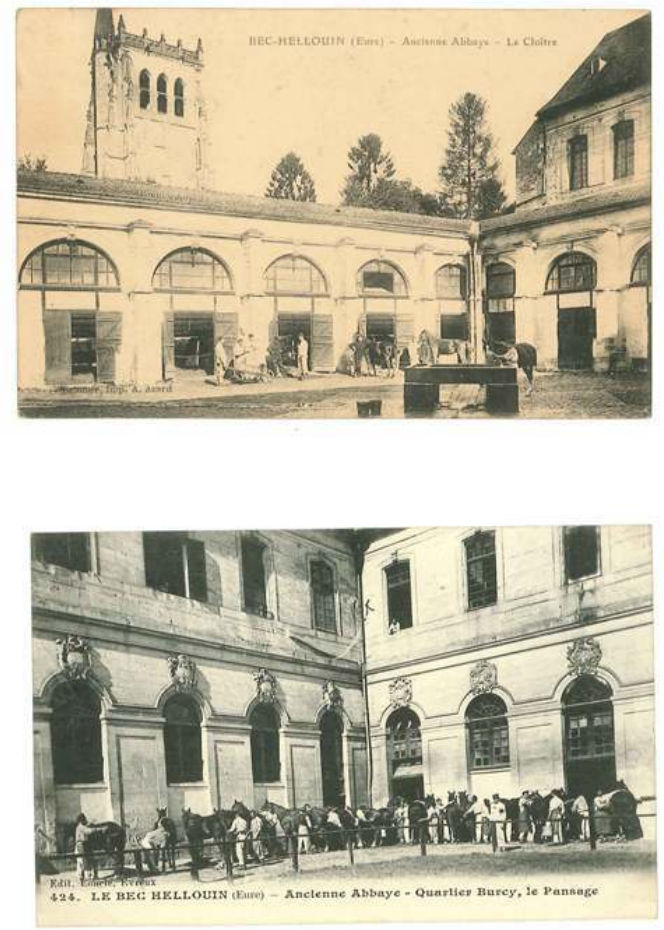

Le Bec-Hellouin (Eure). Ancienne abbaye. En haut : le cloître. En bas : le Quartier Burcy, le pansage. Cartes postales anciennes.

(c) Tanneguy de Sainte Marie.

9 Toujours à cette période napoléonienne, un dépôt d'étalons fut installé à Villeneuved'Agen, déménagé à Libourne, puis enfin à Villeneuve-sur-Lot dans le couvent des Cordeliers dont, de nos jours, il ne reste que la chapelle devenue conciergerie.

En Bretagne, le choix fut très difficile entre l'enclos des Ursulines de Pontivy et l'abbaye cistercienne de Langonnet. C'est finalement cette dernière qui fut retenue et transformée. Elle sera rendue aux religieux Spiritains en 1860 lors du déménagement du dépôt d'étalons à Hennebont (Morbihan), qui plus est, sur le site de son ancienne abbaye !

11 Depuis 1797, confirmé par le décret impérial de 1806, le dépôt d'étalons d'Angers était installé dans l'enclos de l'Hospice des Incurables. Cet établissement fut détruit en 1975 à la suite de son déménagement sur le domaine de L'Isle Briand au Lion d'Angers, qui en a conservé les grilles d'entrée et les piliers surmontés de têtes de chevaux, installés en son entrée de service.

À Saint-Lô, c'est l'important enclos de l'abbaye Sainte-Croix qui abrita un dépôt d'étalons et un dépôt de remonte militaire. Un nouvel établissement avait été construit sur le site actuel en 1880 et l'ancien dépôt "Sainte-Croix" sera entièrement rasé lors des bombardements de juin 1944 (fig . n²). 

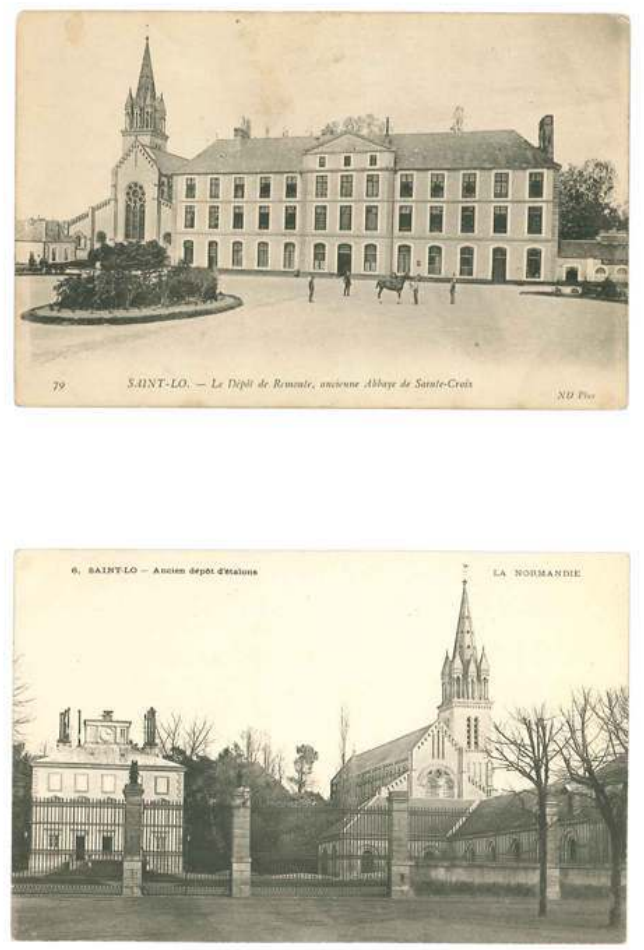

Saint-Lô (Manche). En haut : le dépôt de remonte, ancienne abbaye de Sainte-Croix. En bas : ancien dépôt d'étalons. Cartes postales anciennes.

(c) Tanneguy de Sainte Marie.

13 À Aurillac en 1806, 40 étalons et 14 palefreniers furent hébergés dans le couvent des Visitandines pour desservir les départements du Cantal, du Puy-de-Dôme et de la HauteLoire. Le dépôt d'étalons déménagera dans un établissement neuf à l'extérieur de la ville en 1983. Suite à ce mouvement, lors de l'installation de l'école de musique dans l'ancien manège du haras, le grand nettoyage des murs permit de découvrir les fresques et les peintures de ce qui était à l'origine la chapelle du couvent.

La Chartreuse de Rodez livra son église pour y installer l'écurie $\mathrm{N}^{\circ} 1$ et le bâtiment des hôtes pour les logements des officiers et le bureau du dépôt. Il nous reste encore aujourd'hui l'entrée du XVIII ${ }^{\mathrm{e}}$ siècle, une partie de la cour d'honneur et un bâtiment voûté.

Le dépôt d'étalons de Cluny sera d'abord installé dans les écuries de l'ancienne abbaye saccagée par la Révolution. Dès 1810, après d'énormes travaux de déblaiement et de construction, surgirent des ruines illustres de l'église du monastère bénédictin l'établissement actuel. Ses écuries sont toujours là et les derniers étalons sont encore logés dans des boxes dont un recouvre le tombeau de Pierre Le Vénérable, Prieur réputé de l'abbaye.

À Montier-en-Der, (Monasterium in dervo : Monastère dans la forêt de chênes), après avoir occupé l'abbaye Saint-Berchaire, le dépôt d'étalons fut reconstruit au même endroit avec les matériaux du monastère, à l'exception de l'église abbatiale devenue église paroissiale. Les pierres de taille furent retournées et dorénavant les sculptures apparaissent à l'intérieur des locaux des bâtiments, notamment dans les greniers. 
17 Au centre d'Annecy, c'est le couvent des Dominicains qui accueillera le dépôt d'étalons en 1806. Trois écuries de 15, 5 et 4 étalons seront aménagées au rez-de-chaussée du bâtiment des hôtes, le logement du chef de dépôt au premier étage, ceux de l'agent comptable et des palefreniers au deuxième. Le magasin à fourrage sera installé dans l'église SaintMaurice voisine. Un nouveau dépôt sera construit entre 1880 et 1885 . Il a cessé définitivement son activité en 2007 et un nouvel avenir pour ce site est en cours de négociations.

18 En 1810, sur le portail de la cour d'entrée de l'abbaye Notre-Dame de Saintes, on pouvait lire: "Caserne de l'Abbaye » et sur le portail d'entrée de son église "Écurie pour 63 chevaux ». En 1846, une ordonnance du roi Louis-Philippe permettra la construction d'un nouveau dépôt, plus grand et plus fonctionnel sur son site actuel. Ces travaux seront confiés à l'architecte Victor Fontorbe.

19 En 1810, également, dans sa partie basse, entre le château et la Loire, la ville de Blois mettra à la disposition des Haras impériaux, le couvent des Carmélites. Les boxes aménagés dans les arcades du cloître accueilleront à partir de 1880, après la construction du nouveau haras à l'extérieur de la ville, les chevaux de la gendarmerie qui, aujourd'hui, l'occupe toujours mais avec ses automobiles.

20 En 1860, sous l'impulsion du général Fleury, ministre polyvalent à tout faire de Napoléon III, seront décidés des échanges de parcelles entre le village du Pin (Orne) et le Haras impérial. L'opération doit permettre de reconstruire et déménager les bâtiments communaux (église, école, presbytère, mairie, auberge, boulangerie, etc.) en bordure de la route de Paris. Cette délocalisation du village entraîne la désaffection de l'ancienne église paroissiale qui est alors aménagée en une écurie d'une dizaine de stalles, la réserve à grain et la sellerie étant basées dans la sacristie. Bien que désaffectées, ces installations sont toujours présentes de nos jours.

À l'automne 2009, Bartabas produira un spectacle équestre dans l'église abbatiale désaffectée de Saint-Ouen de Rouen.

Durant ces deux derniers siècles, les étalons et les chevaux de l'État ont sauvé de la destruction nombre de nos abbayes, monastères, églises, couvents et quelques autres établissements plus récents dédiés à ces seules activités. Ils sont aujourd'hui en cours de cessation d'activités. Certes, la mécanisation et la disparition de l'utilisation des bras et des mains, font que ces bâtiments ne sont plus du tout adaptés à l'hébergement des chevaux. Par ailleurs, la disparition de l'étalonnage public va les libérer.

Ce sont donc les loisirs, la culture, l'artisanat, les lettres et les arts complétés des technologies de demain qui doivent y prendre place et les animer.

\section{RÉSUMÉS}

La Révolution française de 1789-1793 a vidé et confisqué les abbayes, les couvents, les monastères et tous les établissements abritant des congrégations religieuses. En y installant des dépôts d'étalons et des dépôts de remonte militaire, Napoléon $1^{\mathrm{er}}$ en a sauvé un certain nombre entre 
1806 et 1814 . De très nombreux autres établissements sont devenus des carrières de pierres et ont disparu. Les évolutions stratégiques, politiques et techniques des armées et des haras nationaux font que certains de ces édifices historiques seront progressivement et à nouveau en grand danger.

INDEX

Mots-clés : abbaye, monastère, couvent, église, étalon

\section{AUTEUR}

\section{TANNEGUY DE SAINTE MARIE}

Ingénieur des haras nationaux, Haras du Pin tanneguy.de_sainte_marie@haras-nationaux.ifce.fr 